\title{
Role of P-Selectin in Platelet Sequestration in Pulmonary Capillaries during Endotoxemia
}

\author{
Rainer Kiefmann ${ }^{\mathrm{a}, \mathrm{b}}$ Kai Heckel ${ }^{\mathrm{a}, \mathrm{b}}$ Sonja Schenkat ${ }^{\mathrm{b}}$ Martina Dörger ${ }^{\mathrm{c}}$ \\ Alwin Eduard Goetz ${ }^{a, b}$ \\ ${ }^{a}$ Department of Anesthesiology, University Medical Center Hamburg-Eppendorf, Hamburg; \\ ${ }^{b}$ Department of Anesthesiology, University of Munich and ${ }^{C}$ Department of Anesthesiology and \\ Institute for Surgical Research, Ludwig Maximilians University, Munich, Germany
}

\section{Key Words}

Platelets · P-selectin · Alveolar capillary networks · Acute

lung injury $\cdot$ Endotoxemia

\begin{abstract}
Background: There is growing evidence that platelets accumulate in the lung and contribute to the pathogenesis of acute lung injury during endotoxemia. The aims of the present study were to localize platelet sequestration in the pulmonary microcirculation and to investigate the role of P-selectin as a molecular mechanism of platelet endothelial cell interaction. Methods: We used in vivo fluorescence microscopy to quantify the kinetics of fluorescently labeled erythrocytes and platelets in alveolar capillary networks in rabbit lungs. Results: Six hours after onset of endotoxin infusion we observed a massive rolling along and firm adherence of platelets to lung capillary endothelial cells whereas under control conditions no platelet sequestration was detected. P-selectin was expressed on the surface of separated platelets which were incubated with endotoxin and in lung tissue. Pretreatment of platelets with fucoidin, a P-selectin antagonist, significantly attenuated the endotoxin-induced platelet rolling and adherence. In contrast, intravenous infusion
\end{abstract}

R. Kiefmann and K. Heckel contributed equally to this study. of fucoidin in endotoxin-treated rabbits did not inhibit platelet sequestration in pulmonary capillaries. Conclusion: We conclude that platelets accumulate in alveolar capillaries following endotoxemia. P-selectin expressed on the surface of platelets seems to play an important role in mediating this platelet-endothelial cell interaction.

Copyright $\odot 2006$ S. Karger AG, Basel

\section{Introduction}

There is growing evidence that platelets play a crucial role in the pathogenesis of acute respiratory distress syndrome and postischemic reperfusion injury following lung transplantation $[1,2]$. Histologic investigations of acute lung injury have shown that platelets sequestrate in pulmonary capillaries prior to neutrophils [3]. Once retained platelets may contribute to tissue damage due to release of oxygen radicals and proinflammatory and vasoactive mediators such as thromboxane $\mathrm{A}_{2}$, leukotrienes, serotonin, platelet factor 4 , and platelet-derived growth factor [4-7]. In addition, platelets have the potential to influence the immunologic response of neutrophils [8]. Therefore, entrapped platelets might promote activation and recruitment of leukocytes at the site of injury and aggravate pulmonary endothelial cell damage.

Prof. Dr. A.E. Goetz

Department of Anesthesiology

University Medical Center Hamburg-Eppendorf, Martinistrasse 52

DE-20246 Hamburg (Germany)

Tel. +49 4042803 2415, Fax +49 4042803 4963, E-Mail a.goetz@uke.uni-hamburg.de 
The mechanisms of pulmonary platelet sequestration might be complex and have not been understood so far. Although platelets are anuclear fragments of megakaryocytes, they possess cellular components enabling their interaction with the endothelium. For instance, platelets carry adhesion molecules required for cell-cell interaction, such as PECAM-1, integrins (LFA-1, glycoprotein IIb/IIIa), and P-selectin $[9,10]$. The last of these is stored in $\alpha$-granule of platelets and in Weibel-Palade bodies of endothelial cells. Upon activation of these cells P-selectin is expressed on the cell surface within seconds and mediates rolling of leukocytes along the endothelium. There is growing evidence that $\mathrm{P}$-selectin is also responsible for the initial interaction between activated platelets and endothelium, since rolling of platelets has also been observed [11]. However, the role of P-selectin in the mediation of platelet/endothelial interaction in pulmonary capillaries is completely unknown.

Therefore, the aim of the study was to characterize the behavior of platelets in pulmonary capillary networks under physiologic conditions and after endotoxemia in vivo using intravital fluorescence microscopy. The role of endothelial and/or platelet P-selectin in platelet/endothelial cell interaction was investigated by either intravenous administration of the P-selectin antagonist fucoidin or by incubation of fluorescent-labeled platelets with fucoidin prior to reinjection.

\section{Methods}

\section{Animal Preparation}

All animals received care in accordance with the German legislation on protection of laboratory animals. The anesthesia protocol, the surgical preparation and experimental setup have been described in detail [12]. In brief, male New Zealand White rabbits ranging in body weight (BW) from 2.5 to $3.3 \mathrm{~kg}$ were anesthetized, tracheotomized, intubated and pressure-controlled ventilated $\left(\mathrm{FiO}_{2}: 0.4\right.$, inspiratory airway pressure $8 \mathrm{~mm} \mathrm{Hg}$, expiratory airway pressure $2 \mathrm{~mm} \mathrm{Hg}$ ). For continuous monitoring of macrohemodynamics catheters were introduced into the carotid and pulmonary artery and into the central vein. After implantation of a transparent window into the right chest wall in vitro fluorescein isothiocyanate (FITC)-labeled red blood cells (Sigma, Deisenhofen, Germany) and in vitro rhodamine 6G-labeled platelets (Merck, Darmstadt, Germany) were sequentially visualized in subpleural alveolar capillary networks during prolonged inspiration periods of $10 \mathrm{~s}$ using a fluorescence microscope (Leica, Wetzlar, Germany). For video recordings a silicon-intensified video camera (C2400-08, Hamamatsu, Herrsching, Germany) and an S-VHS video recorder (AG-7350, Panasonic, Munich, Germany) were used.

\section{Cell Labeling}

Red blood cells harvested from carotid artery were separated by centrifugation and labeled with FITC as previously described [12]. The FITC-labeled red blood cells were resuspended in saline solution and reinfused. Platelets were prepared according to Massberg et al. [11]. Briefly, $10 \mathrm{ml}$ of whole blood were mixed with $3 \mathrm{ml}$ phosphate-buffered saline (PBS) and $0.55 \mathrm{ml}$ aqua ad injectabilia containing $15.2 \mu \mathrm{mol}$ citric acid, $30 \mu \mathrm{mol}$ trisodium citrate, $40 \mu \mathrm{mol}$ dextrose, and $3 \mu \mathrm{g} \mathrm{PGE}_{1}$. Blood was centrifuged and platelets were separated. Fifteen microliters of the $0.05 \%$ fluorescent dye rhodamine $6 \mathrm{G}$ per $1 \mathrm{ml}$ platelet-rich plasma were added. After centrifugation $(2,000 \mathrm{~g} ; 10 \mathrm{~min})$, separation and resuspendation with $10 \mathrm{ml}$ PBS half of the platelets were incubated with $3 \mathrm{mg}$ of the sulfated fucose polymer fucoidin (Sigma, Deisenhofen, Germany) and centrifuged again. Platelets were resuspended with $2 \mathrm{ml} \mathrm{PBS}\left(0.5 \cdot 10^{9}\right.$ platelets $\left./ \mathrm{ml}\right)$ and reinjected shortly before intravital microscopy was performed.

\section{Determination of P-Selectin Expression by}

Immunohistochemistry/Cytochemistry

Paraffin-embedded lung sections and cytospins of separated platelets were stained with the primary rabbit-specific monoclonal anti-P-selectin antibody Psel.KO2.10 (1:50 dilution) and with the ABC kit (Dianova, Hamburg, Germany) according to the manufacturer's instructions. Psel.KO2.10 was a generous gift from Pilar Pizcueta (Servicio de Cardiologia, Hospital Universitari Vall d'Hebron, Barcelona, Spain).

\section{Determination of Expression of P-Selectin on Isolated}

Platelets by Flow Cytometry

Isolated rabbit platelets were incubated for $10 \mathrm{~min}$ with a crossreacting FITC-stained anti-C62P antibody (Ms IgG1 Clone AK-4 mouse anti-human CD62P, FITC, Acris Diagnostika, Hiddenhausen, Germany) at a final concentration of $20 \mu \mathrm{l} / 1.0 \cdot 10^{9}$ platelets. For negative control a nonreacting FITC-stained mouse IgG1 antibody was used. Samples were washed twice with PBS to minimize autofluorescent activity of fucoidin and analyzed by flow cytometry (FACScan cytometer, Becton Dickinson, Heidelberg, Germany).

Determination of Microhemodynamics, Platelet-Endothelial

Interaction by Intravital Fluorescence Microscopy

Microhemodynamics and platelet kinetics were analyzed offline from the video recordings using a digital image-processing system (Optimas, Bioscan, Edmonds, Wach., USA), as described previously $[12,13]$. The inner wall of the alveoli was outlined and the respective alveolar surface area was planimetrically determined by the image analysis system. Microhemodynamics and platelet kinetics were described as the harmonic mean of the velocity of at least 30 FITC-labeled erythrocytes and rhodamine 6G-labeled platelets passing a predefined vessel cross section or capillary network. Adherent platelets were defined as cells not moving for $>10 \mathrm{~s}$ and expressed per projected alveolar surface, respectively. At least three different alveolar areas were investigated in each experimental phase.

\section{Experimental Protocol}

To investigate the effect of endotoxemia on platelet/endothelium interaction intravital microscopy was performed under control conditions and after a 6-hour endotoxin infusion. The ani- 
mals were randomly treated with a saline solution $(\mathrm{n}=5 ; 2 \mathrm{ml}$ as bolus and $1 \mathrm{ml} / \mathrm{h} \cdot \mathrm{kg} \mathrm{BW}$; per infusionem) or with endotoxin ( $\mathrm{n}=5$; LPS 0111:B4 from Escherichia coli, Sigma). First, $100 \mu \mathrm{g}$ of LPS were administered as bolus followed by an LPS infusion $(20 \mu \mathrm{g} / \mathrm{h} \cdot \mathrm{kg} \mathrm{BW})$ until the end of the experiment. Substances were delivered through the central vein catheter. Macrohemodynamics was monitored continuously. After surgical preparation of the animals and confirming of the exclusion criteria, as mean arterial pressure $<65 \mathrm{~mm} \mathrm{Hg}$, lack of macroscopic visible atelectasis, hemorrhage, or perfusion failure on lung surface, autologous FITC-labeled erythrocytes were reinjected $30 \mathrm{~min}$ before intravital microscopy ensuring splenic elimination of rheologically altered labeled red blood cells. Rhodamine 6G-labeled platelets were reinfused immediately before the video microscopic pictures were taken. For endothelial P-selectin inhibition $20 \mathrm{mg} / \mathrm{kg}$ BW of fucoidin was intravenously administered $15 \mathrm{~min}$ before platelet reinfusion. To prevent anaphylactic reactions induced by fucoidin $0.3 \mathrm{mg} / \mathrm{kg}$ BW of monovalent dextran (molecular weight 1,000 ; Promit ${ }^{\circledR}$, Schiwa, Glandorf, Germany) was given 3 min before the infusion of fucoidin. To evaluate the effect of platelet P-selectin inhibition on platelet/endothelial cell interaction, isolated rhodamine $6 \mathrm{G}$-labeled platelets $\left(0.5 \cdot 10^{9} / \mathrm{ml}\right.$ buffer $)$ were incubated with either LPS $(300 \mathrm{ng} / \mathrm{ml})$ or LPS $(300 \mathrm{ng} / \mathrm{ml})$ and fucoidin $(200 \mathrm{mg} / \mathrm{ml})$, washed, and reinfused for intravital microscopy. At the end of the experiments, the animals were sacrificed and the lungs removed for immunohistochemistry. A portion of platelets prepared for intravital microscopy were separated and used for immunocytochemistry.

\section{Statistics}

All data are represented as mean \pm standard error of the mean. Statistical data analysis was performed using SigmaStat (Jandel, 1995, Erkrath, Germany). Comparisons between the groups were tested using ANOVA on ranks followed by Dunn's method. Repeated measurements were tested using the Mann-Whitney rank sum test. Statistical significance was assumed when $\mathrm{p}<0.05$.

\section{Results}

\section{Expression of P-Selectin on Isolated Platelets Detected} by Immunocytochemistry

Anti-P-selectin antibody binding on control platelets was not detectable indicating that under these conditions platelet P-selectin expression was not expressed or below our detection limit. Incubation of platelets with LPS for 1 min markedly enhanced immunoreactivity for P-selectin. The additional incubation of platelets with fucoidin

Fig. 1. Immunocytochemical staining of P-selectin (arrows) on platelet under control conditions (a), after LPS (b), and after LPS + fucoidin (c). Platelets treated with LPS revealed intensive expression of P-selectin. In contrast, pretreatment with fucoidin inhibited LPS-induced P-selectin staining. Experiments were repeated 4 times.
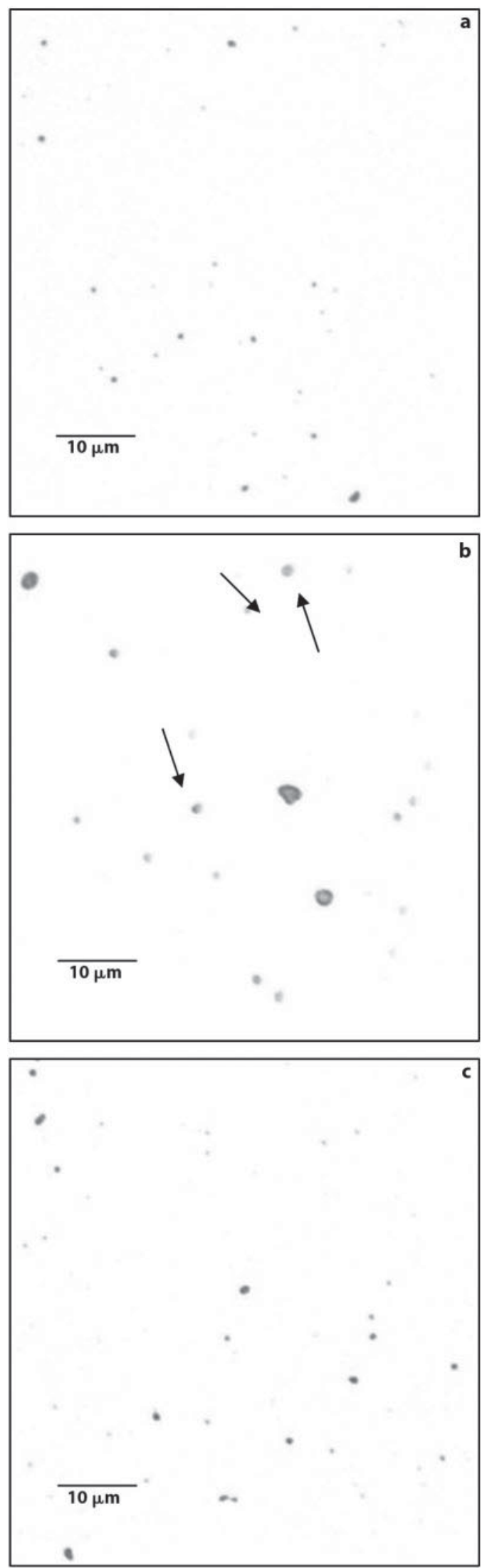


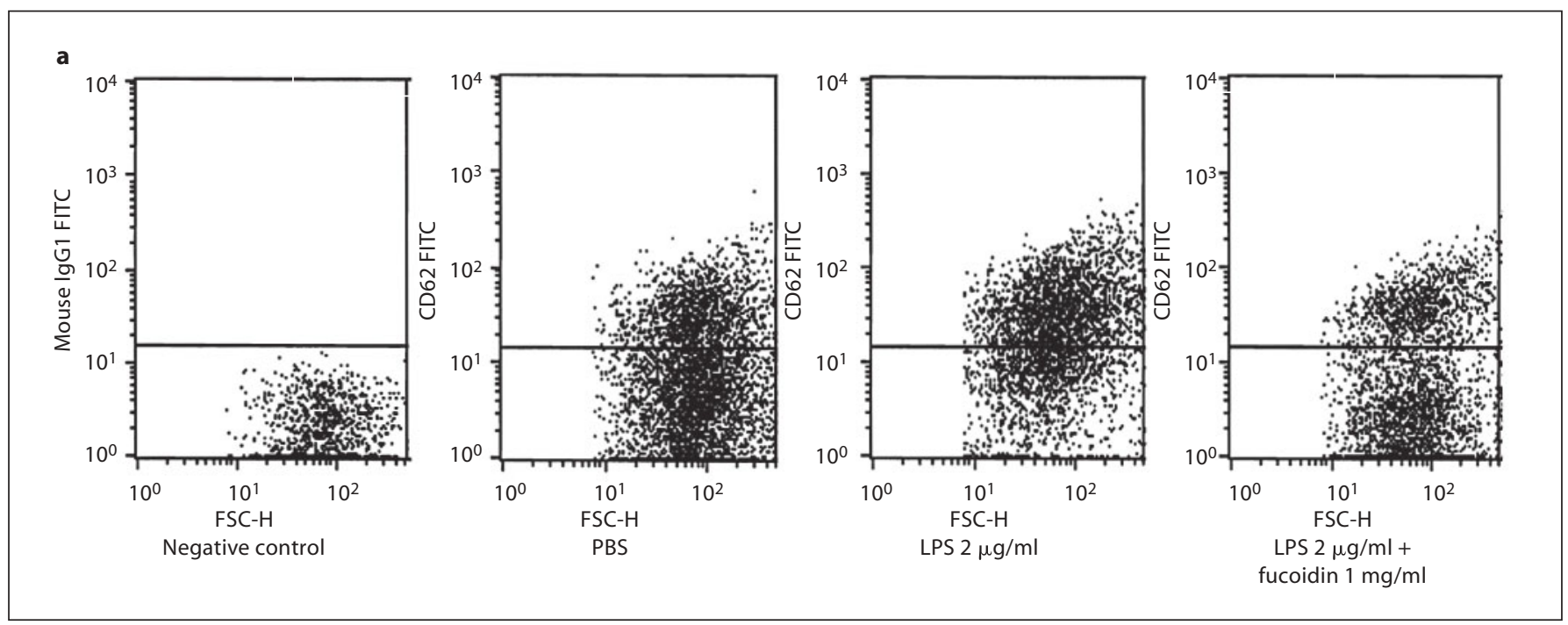

Fig. 2. a Flow cytometry analysis of P-selectin expression of isolated platelets. Platelets were treated with nonreacting FITCstained mouse IgG1 antibody as negative control, or with PBS, LPS $2 \mu \mathrm{g} / \mathrm{ml}$, and LPS $2 \mu \mathrm{g} / \mathrm{ml}+$ fucoidin $1 \mathrm{mg} / \mathrm{ml}$. Treatment of platelets with LPS revealed increased expression of P-selectin. In contrast, addition of fucoidin decreased LPS-induced P-selectin staining. b Flow cytometry group data analysis of platelet P-selectin expression. Percentage of P-selectin-positive platelets after incubation with PBS, PBS + fucoidin $1 \mathrm{mg} / \mathrm{ml}$, LPS $2 \mu \mathrm{g} / \mathrm{ml}$, and LPS $2 \mu \mathrm{g} / \mathrm{ml}+$ fucoidin $1 \mathrm{mg} / \mathrm{ml}$. Data are mean \pm SEM, $\mathrm{n}=4$.

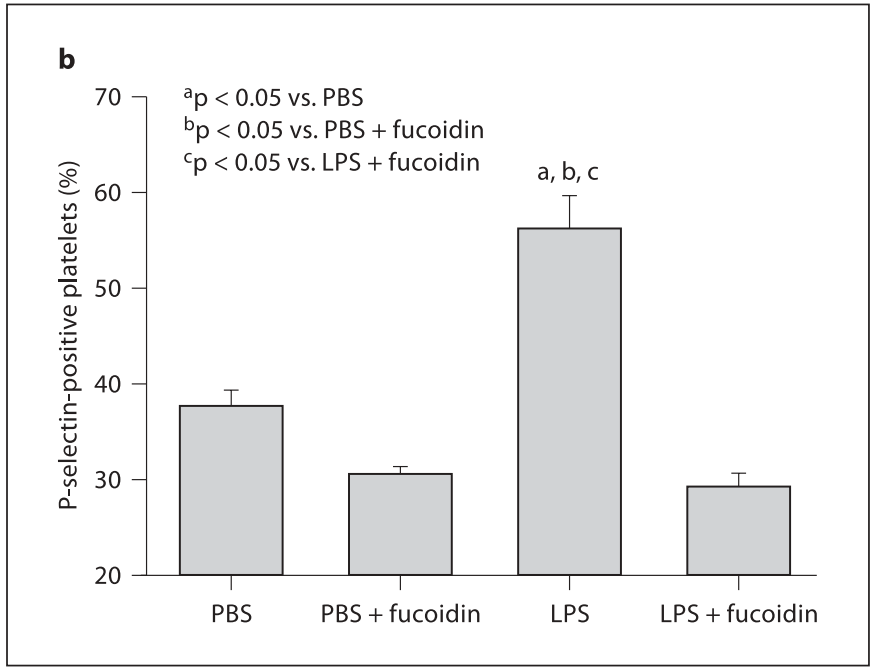

blocked the antibody binding to P-selectin nearly completely (fig. 1). Similar results were obtained with thrombin $(1 \mathrm{U} / \mathrm{ml})$ instead of LPS (two experiments, data not shown).

\section{Expression of P-Selectin on Isolated Platelets Detected} by Flow Cytometry

In each platelet suspension $6,960 \pm 726$ platelets were analyzed. Anti P-selectin antibody binding was found on $37.6 \pm 1.5 \%$ of PBS-treated platelets. However, incubation of platelets with LPS significantly enhanced P-selectin expression (56.2 $\pm 3.0 \%$ of platelets). In contrast, this response was completely blocked by fucoidin (LPS + fucoidin: $29.2 \pm 1.2 \%$ of platelets, PBS + fucoidin: $30.5 \pm$ $0.7 \%$ of platelets) (fig. $2 a, b$ ).

\section{Expression of P-Selectin in Lung Tissue}

Only small amounts of P-selectin could be detected under control conditions on the inner surface of blood vessels, whereas $6 \mathrm{~h}$ after LPS infusion the staining intensity increased. The antibody binding to P-selectin on the pulmonary endothelium was completely inhibited by the administration of fucoidin (fig. 3).

\section{Microhemodynamics}

Microhemodynamics and platelet kinetics were quantified in the identical alveolar capillary network, three per animal. There was no difference of the alveolar wall area between the control $\left(10.2 \cdot 10^{3} \mu \mathrm{m}^{2}\right)$ and LPS group $\left(8.9 \cdot 10^{3} \mu \mathrm{m}^{2}\right)$. The mean erythrocyte velocity in the control group was $628 \pm 18 \mu \mathrm{m} / \mathrm{s}$ and in the LPS group 760 

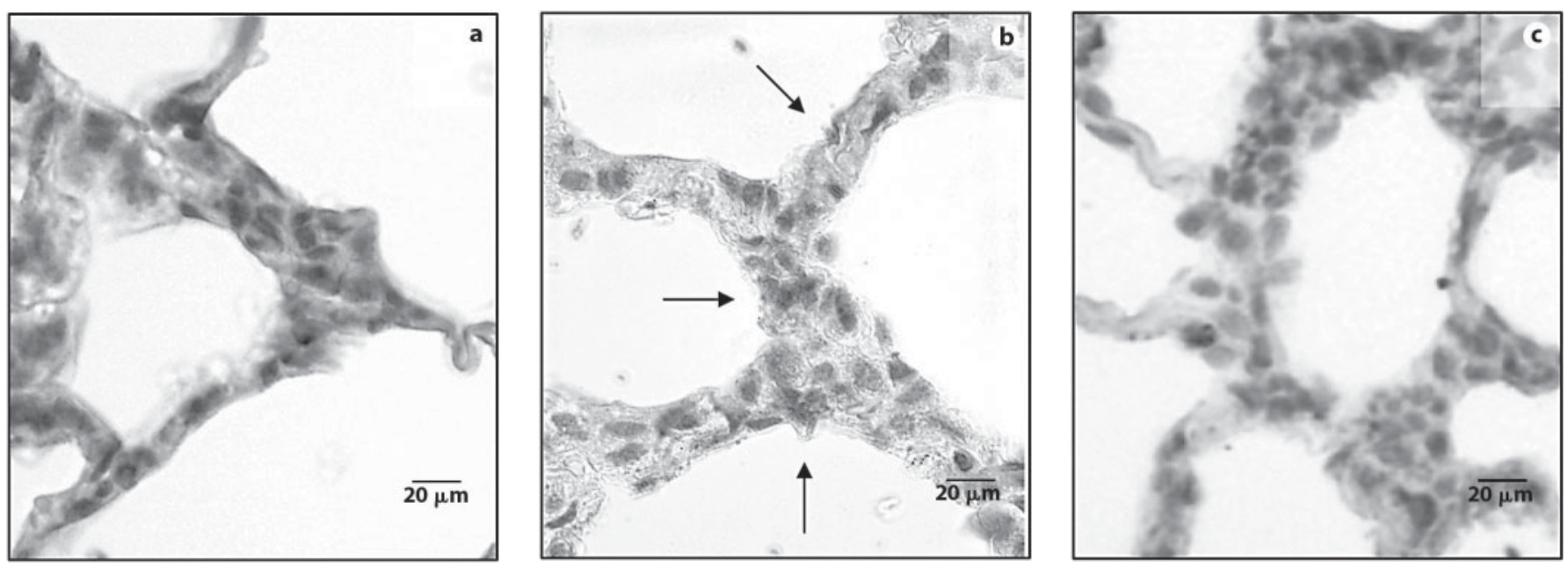

Fig. 3. Immunohistochemical staining of P-selectin (arrows) in lung sections under control conditions (a), after LPS (b), and after LPS + fucoidin (c). Animals treated with LPS but not those from control group showed intensive expression of P-selectin. However, pretreatment of the animals with fucoidin completely inhibited the P-selectin staining induced by LPS infusion. Experiments were repeated 4 times.

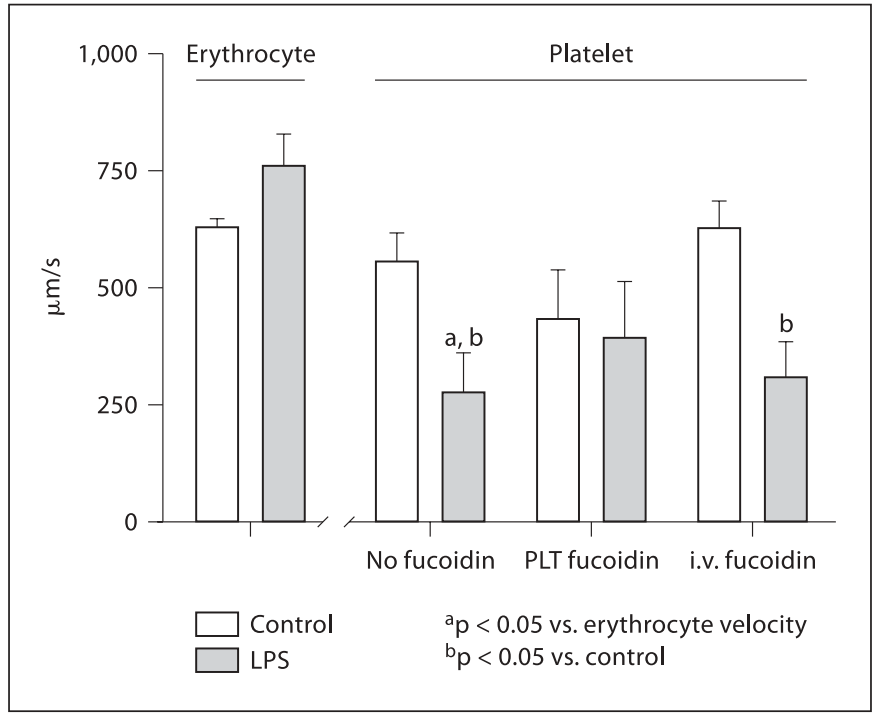

Fig. 4. Velocity of erythrocytes and platelets (PLT) in pulmonary alveolar capillary networks $6 \mathrm{~h}$ after saline $(\mathrm{n}=4)$ and LPS $(\mathrm{n}=$ 5). Untreated platelets or fucoidin-treated platelets were investigated before and after systemic administration of fucoidin. Data are mean \pm SEM.

$\pm 68 \mu \mathrm{m} / \mathrm{s}$ and did not statistically differ between the two groups (fig. 4). Infusion of fucoidin did not influence the red blood cell velocity either in the control or in the LPS group (data not shown).

P-Selectin and Pulmonary Platelet Sequestration

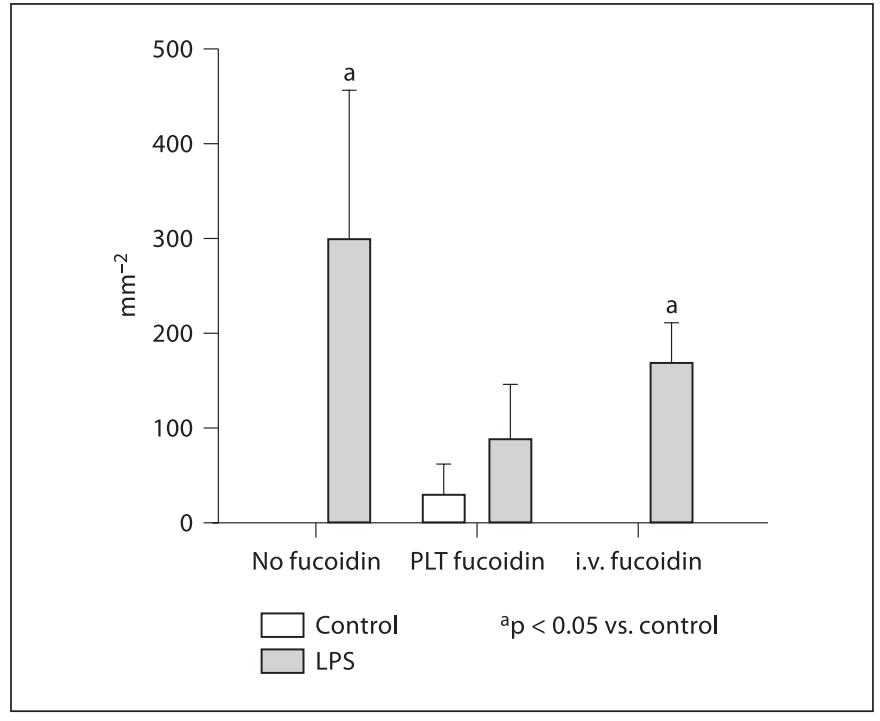

Fig. 5. Number of sticking platelets (PLT) per area in pulmonary alveolar capillary networks $6 \mathrm{~h}$ after saline $(\mathrm{n}=4)$ and LPS $(\mathrm{n}=$ 5). Untreated platelets or fucoidin-treated platelets were investigated before and after systemic administration of fucoidin. Data are mean \pm SEM.

\section{Platelet-Endothelial Interaction}

In alveolar capillary networks untreated platelets (not incubated with fucoidin) had the same mean velocity (555 $\pm 62 \mu \mathrm{m} / \mathrm{s}$ ) as erythrocytes and were not perma-

J Vasc Res 2006;43:473-481 
nently retained (sticking), indicating that unstimulated platelets and erythrocytes possess an equal rheological behavior. In contrast, infusion of LPS resulted in a significant decrease of the mean platelet velocity (276 \pm $85 \mu \mathrm{m} / \mathrm{s}$ ) and an increase of the permanently retained platelets $\left(299 \pm 157 \mathrm{~mm}^{-2}\right)$ and therefore in a massive retention of platelets in alveolar capillaries versus control. Under control conditions the incubation of platelets with fucoidin and the systemic administration of fucoidin did not significantly influence either the mean platelet velocity (fucoidin-treated platelet velocity: $432 \pm 106 \mu \mathrm{m} / \mathrm{s}$; platelet velocity after systemic fucoidin: $626 \pm 58 \mu \mathrm{m} / \mathrm{s}$ ) or the number of sticking platelets in alveolar capillaries $\left(28 \pm 33\right.$ and $0.0 \pm 0.0 \mathrm{~mm}^{-2}$, respectively). However, the pretreatment of platelets with fucoidin inhibited the LPS-induced decrease of mean platelet velocity (392 \pm $121 \mu \mathrm{m} / \mathrm{s}$ ) and the increase of permanently retained platelets $\left(88 \pm 56 \mathrm{~mm}^{-2}\right)$ since there was no significant difference from the control. In comparison, the intravenous infusion of fucoidin had no inhibitory effect on platelet retention after LPS in pulmonary capillary networks (platelet velocity: $308 \pm 75 \mu \mathrm{m} / \mathrm{s}$, number of sticking platelets: $168 \pm 42 \mathrm{~mm}^{-2}$ ) (fig. 4,5 ). The number of permanently retained platelets following LPS did not statistically differ between the groups.

\section{Discussion}

The main finding of our study was that intravenous infusion of endotoxin caused a massive accumulation of platelets in the alveolar capillary network within minutes after their reinjection. Experiments were performed using differently in vitro fluorescence-labeled erythrocytes and platelets. The staining technique used for the study has already been validated for the investigation of platelet kinetics in a mouse small intestine model following ischemia/reperfusion [11]. The mean platelet velocity was decreased and platelet aggregates were observed within the pulmonary capillaries as a sign of a temporary interaction of platelets with the endothelium. Macro- and microhemodynamics did not change following the infusion of endotoxin. Shear-dependent mechanisms as a possible cause of enhanced platelet endothelial interactions can therefore be ruled out. It has previously been reported that endothelial P-selectin [14] may promote binding of erythrocytes to endothelial cells and, thus, affect microhemodynamics. Obviously, this appears to play a minor role in the rabbit pulmonary microcirculation, since erythrocyte velocities did not change following LPS infusion.
Since the observed platelet retention within septal capillaries following endotoxin infusion was not caused by changes of microhemodynamics we tested whether P-selectin might be involved in this platelet/endothelial cell interaction.

P-selectin, a lectin-like molecule which mediates the initial step of the adhesion cascade (rolling), is stored in intracellular granules of platelets and endothelial cells $[15,16]$. Immediately upon activation this adhesion molecule can be mobilized to the cell surface independent of gene transcription and expression [17, 18]. Using immunocytochemistry and flow cytometry we demonstrated that after activation with endotoxin P-selectin was expressed within minutes on the surface of platelets. There has already been a study on the potential of endotoxin to induce in vivo $\mathrm{P}$-selectin translocation in circulating platelets [19]. We were also able to detect P-selectin expression in lung tissue. Immunostaining of P-selectin on tissue sections detects both intracellular and surface-expressed P-selectin. It can be considered that following cell activation and translocation of P-selectin to the cell surface the total amount of P-selectin protein per cell remains more or less unchanged. Therefore, a local enhancement of P-selectin staining could be due to accumulated platelets. In our study, lungs were removed for immunohistochemistry $6 \mathrm{~h}$ after the onset of LPS infusion. It has been demonstrated that P-selectin transcription in endothelial cells can be upregulated within $6 \mathrm{~h}$ following LPS stimulation [20]. Platelets do not have a nucleus and, thus, P-selectin upregulation in these cells is unlikely. Therefore, the enhanced immunostaining of P-selectin following LPS, as detected in our study, can be caused not only by attached platelets but also by endothelial P-selectin upregulation. Although we were not able to demonstrate P-selectin mobilization to the endothelial cell surface by immunohistochemistry studies, both in vitro and ex vivo experiments have previously documented that LPS induces rapid mobilization of P-selectin on the endothelial cell surface [20].

We evaluated the role of P-selectin in mediating the initial step of platelet-endothelial interactions in pulmonary septal capillaries upon infusion of endotoxin using the P- and L-selectin antagonist fucoidin. According to Handa et al. [21] sulfate glycans such as fucoidin may inhibit the binding of EGF-like selectin domains with its ligands by inducing conformational changes of this domain. Fucoidin was shown to be a potent inhibitor of leukocyte rolling by blocking P- and L-selectin in a dose-dependent manner [22]. However, Kubes et al. [23] proposed the existence of fucoidin sensitive but L- and 
P-selectin-independent leukocyte rolling. They demonstrated that the inhibition of L- and P-selectin with two different antibodies only reduced leukocyte rolling in venules upon ischemia/reperfusion by $60 \%$, whereas fucoidin induced a $90 \%$ reduction of leukocyte rolling. The proposed existence of selectin-unspecific effects of fucoidin on platelet kinetics has not been investigated so far and can therefore not be excluded in the present study. It should be considered that the binding of fucoidin may activate platelets and therefore alter their kinetics. However, neither the pretreatment of platelets with fucoidin nor the infusion of fucoidin did alter the platelet velocity compared to control conditions. Toxic or anaphylactic side effects of fucoidin also seem to be unlikely since none of the parameters assessed in the present study significantly changed after the infusion of fucoidin.

Pretreatment of platelets with fucoidin dramatically attenuated rolling and subsequent firm adhesion to capillary endothelium after endotoxin. In contrast, infusion of fucoidin did not influence the endotoxin-induced changes of platelet kinetics in alveolar capillaries. The binding of fucoidin on rabbit platelets and lung endothelium was confirmed by previous studies and by immunohistochemistry. We demonstrated in a comparable rabbit model that the infusion of fucoidin significantly increased the flow velocity of moving leukocytes, reduced frequency and duration of temporary leukocyte retention, drastically accelerated the leukocyte transit in alveolar capillaries [24], and inhibited the endotoxin-induced decrease of platelet velocity in pulmonary arterioles and venules [25]. In the present study, P-selectin expression was found in rabbit lungs and separated platelets treated with endotoxin alone and could not be determined in rabbit lungs or platelets treated with endotoxin and fucoidin. We could not differentiate whether the endotoxin-induced pulmonary expression of P-selectin was due either to platelet adhesion or to redistribution of P-selectin on the endothelial cell surface. However, it is published that, for example, thrombin which is generated during endotoxemia induces mobilization of P-selectin on the endothelial cell surface. Our results provide therefore strong evidence that P-selectin expressed by the platelet is the major determinant responsible for the platelet sequestration in pulmonary capillary networks following endotoxin. The endothelial P-selectin seems to play only a minor role in mediating platelet-endothelial cell interaction in our model.

This finding is partially in contrast to our data published in Thrombosis and Haemostasis in 2004 [25]. In this study we found that intravenous infusion of endotoxin re- sulted in a decrease of platelet velocity in pulmonary arterioles and venules and this effect could be partly inhibited by intravenous infusion of fucoidin. From this result we concluded that endothelial P-selectin is involved in the interaction of platelets with the endothelium and that, for example, other adhesion molecules such as E-selectin might be involved in this process. The finding that endothelial P-selectin seems to be involved in pulmonary arterioles and venules but not in capillaries can be explained by the publication of Feuerhake et al. [26]. They detected an endothelial P-selectin expression in pulmonary arterioles and venules but not in septal capillaries. Our results go also along with the findings of Kubo et al. [27]. They observed that the intravenous infusion of fucoidin but not anti P-selectin antibodies blocked the complement fragment-induced leukocyte sequestration in pulmonary capillaries. Since on the one hand endothelial cells but not leukocytes express P-selectin following adequate stimulation and on the other hand leukocytes possess ligands for P-selectin and express L-selectin which can also be blocked by fucoidin, the authors concluded that leukocyte sequestration in pulmonary capillaries is mediated by the leukocyte L-selectin rather than by the endothelial P-selectin. One can also conclude from these findings that endothelial cells in pulmonary capillaries must express a ligand for L-selectin. As summarized by Rinko et al. [28] structural similarities between E-, P-, and L-selectin suggest that they bind similar or even identical ligands. In fact, all three selectins recognize and bind to sialyl Lewis (sLe), a rigid polysaccharide, in particular, sLex and sLea [29-31]. Taken together, according to Kubo et al. pulmonary capillary endothelial cells express a ligand for L-selectin and according to Rinko et al. P- and L-selectin recognize the identical ligand. Therefore, $\mathrm{P}$-selectin-expressing platelets should similarly to L-selectin-expressing leukocytes be able to interact with the endothelium of pulmonary capillaries and the blockade of platelet P-selectin should according to our findings reduce the platelet/endothelial interaction following stimulation.

In the case of inflammation heparan sulfates are also potential ligands for $\mathrm{P}$ - and L-selectin as reviewed by Parish [32]. Heparan sulfates are ubiquitously expressed polysaccharides on cell surfaces and in cell matrix. It has been demonstrated by Wang et al. [33] that endothelial heparane sulfate can act as the dominant L-selectin ligand on inflamed vascular endothelium. Since endothelial heparane sulfates ligate also P-selectin it is theoretically possible that platelets interact with the endothelium by platelet P-selectin/endothelial heparan sulfate binding. As far as we know, this hypothesis has not been test- 
ed. However, it could explain why in our study the platelet $\mathrm{P}$-selectin inhibition with fucoidin reduced the endotoxin-induced platelet endothelial interaction.

Nevertheless there are numerous in vivo studies showing that endothelial, rather than platelet P-selectin mediates platelet endothelium interactions in vivo. Indeed, there are studies, e.g. by Massberg et al. [11], showing that 'platelets lacking P-selectin rolled along or adhered to postischemic wild-type endothelium, interactions between wild-type platelets with mutant endothelium were nearly absent, indicating that I/R-induced platelet-endothelium interactions are dependent on the expression of P-selectin by endothelial cells'. However, under certain conditions platelet P-selectin seems to mediate platelet/ endothelial interaction: Frenette et al. [34] have demonstrated numerous interactions of activated platelets from wild-type mice in TNF- $\alpha$-stimulated venules of $\mathrm{P} / \mathrm{E}-/-$ mice, whereas very little capture and rolling were seen when activated P-selectin-/- platelets were injected or when mice were not treated with TNF- $\alpha$.

Since TNF- $\alpha$ seems to be important for platelet P-selectin-mediated platelet/endothelial interaction and TNF- $\alpha$ blood concentration rises during endotoxemia [35] our results are in accordance with the literature. Our concept that platelet P-selectin may contribute to platelet/endothelial interaction might be further supported by Tailor and Granger [36]. They reported: 'When WT platelets were monitored in high cholesterol diet (HCD)-P- selectin-/- mice, platelet/endothelial adhesion was dramatically reduced. However, when P-selectin-/- platelets were monitored in HCD-WT recipients, platelet/endothelial adhesive interactions were reduced even further, comparable to no diet WT mice.'

In conclusion, we have demonstrated that platelets, similar to leukocytes, become sequestrated in alveolar capillary networks in response to endotoxemia. In contrast to leukocytes, platelets do not seem to be retained in pulmonary capillaries due to mechanical hindrance. The platelet accumulation is induced by platelet-endothelial cell interaction and it seems to be mediated via P-selectin expressed on the surface of platelets. Because platelets may release proinflammatory chemokines and oxygen radicals and modulate leukocyte sequestration, platelet accumulation in the pulmonary microvasculature might contribute to lung injury in response to entotoxemia.

\section{Acknowledgments}

The authors thank Prof. Dr. Drs. h.c. mult. K. Messmer and Prof. Dr. Drs. h.c. K. Peter for their generous support. The technical assistance of Alke Schropp, Silvia Münzing and Anne-Marie Allmeling is gratefully acknowledged.

Special thanks got to Dr. B. Fehse (Department of Bone Marrow Transplantation, University Medical Center Hamburg-Eppendorf, Germany) for technical accomplishment of the flow cytometry analysis.

\section{References}

1 Heffner JE, Sahn SA, Repine JE: The role of platelets in the adult respiratory distress syndrome. Culprits or bystanders? Am Rev Respir Dis 1987;135:482-492.

2 Okada Y, Marchevsky AM, Zuo XJ, Pass JA, Kass RM, Matloff JM, Jordan SC: Accumulation of platelets in rat syngeneic lung transplants: a potential factor responsible for preservation-reperfusion injury. Transplantation 1997;64:801-806.

3 Barry BE, Crapo JD: Patterns of accumulation of platelets and neutrophils in rat lungs during exposure to 100 and $85 \%$ oxygen. Am Rev Respir Dis 1985;132:548-555.

4 Leo R, Pratico D, Iuliano L, Pulcinelli FM, Ghiselli A, Pignatelli P, Colavita AR, FitzGerald GA, Violi F: Platelet activation by superoxide anion and hydroxyl radicals intrinsically generated by platelets that had undergone anoxia and then reoxygenated. Circulation 1997;95:885-891.
5 Forde RC, Fitzgerald DJ: Reactive oxygen species and platelet activation in reperfusion injury. Circulation 1997;95:787-789.

6 Piccardoni P, Evangelista V, Piccoli A, de Gaetano G, Walz A, Cerletti C: Thrombinactivated human platelets release two NAP-2 variants that stimulate polymorphonuclear leukocytes. Thromb Haemost 1996;76:780785.

7 Deuel TF, Senior RM, Chang D, Griffin GL, Heinrikson RL, Kaiser ET: Platelet factor 4 is chemotactic for neutrophils and monocytes. Proc Natl Acad Sci USA 1981;78:45844587.

8 Ruf A, Patscheke H: Platelet-induced neutrophil activation: platelet-expressed fibrinogen induces the oxidative burst in neutrophils by an interaction with CD11C/CD18. Br J Haematol 1995;90:791-796.

9 Body SC: Platelet activation and interactions with the microvasculature. J Cardiovasc Pharmacol 1996;27(suppl 1):S13-S25.
10 Williams MJ, Du X, Loftus JC, Ginsberg MH: Platelet adhesion receptors. Semin Cell Biol 1995;6:305-314.

11 Massberg S, Enders G, Leiderer R, Eisenmenger S, Vestweber D, Krombach F, Messmer K: Platelet-endothelial cell interactions during ischemia/reperfusion: the role of $\mathrm{P}$ selectin. Blood 1998;92:507-515.

12 Kuhnle GE, Kuebler WM, Groh J, Goetz AE: Effect of blood flow on the leukocyte-endothelium interaction in pulmonary microvessels. Am J Respir Crit Care Med 1995;152: 1221-1228.

13 Kuhnle GE, Kiefmann R, Sckell A, Kuebler WM, Groh J, Goetz AE: Leukocyte sequestration in pulmonary microvessels and lung injury following systemic complement activation in rabbits. J Vasc Res 1999;36:289298. 
14 Matsui NM, Borsig L, Rosen SD, Yaghmai M, Varki A, Embury SH: P-selectin mediates the adhesion of sickle erythrocytes to the endothelium. Blood 2001;98:1955-1962.

15 Bevilacqua MP, Nelson RM: Selectins. J Clin Invest 1993;91:379-387.

16 Bevilacqua MP: Endothelial-leukocyte adhesion molecules. Annu Rev Immunol 1993; 11:767-804.

17 Stenberg PE, McEver RP, Shuman MA, Jacques YV, Bainton DF: A platelet alphagranule membrane protein (GMP-140) is expressed on the plasma membrane after activation. J Cell Biol 1985;101:880-886.

18 Hattori R, Hamilton KK, McEver RP, Sims PJ: Complement proteins C5b-9 induce secretion of high molecular weight multimers of endothelial von Willebrand factor and translocation of granule membrane protein GMP-140 to the cell surface. J Biol Chem 1989;264:9053-9060.

19 Li N, Soop A, Sollevi A, Hjemdahl P: Multicellular activation in vivo by endotoxin in humans - limited protection by adenosine infusion. Thromb Haemost 2000;84:381387.

20 Dore M, Sirois J: Regulation of P-selectin expression by inflammatory mediators in canine jugular endothelial cells. Vet Pathol 1996;33:662-671.

21 Handa K, Nudelman ED, Stroud MR, Shiozawa T, Hakomori S: Selectin GMP-140 (CD62; PADGEM) binds to sialosyl-Le(a) and sialosyl-Le(x), and sulfated glycans modulate this binding. Biochem Biophys Res Commun 1991;181:1223-1230.
22 Ley K, Linnemann G, Meinen M, Stoolman LM, Gaehtgens P: Fucoidin, but not yeast polyphosphomannan PPME, inhibits leukocyte rolling in venules of the rat mesentery. Blood 1993;81:177-185.

23 Kubes P, Jutila M, Payne D: Therapeutic potential of inhibiting leukocyte rolling in ischemia/reperfusion. J Clin Invest 1995;95: 2510-2519.

24 Kuebler WM, Kuhnle GE, Groh J, Goetz AE: Contribution of selectins to leucocyte sequestration in pulmonary microvessels by intravital microscopy in rabbits. J Physiol 1997;501:375-386.

25 Kiefmann R, Heckel K, Schenkat S, Dorger M, Wesierska-Gadek J, Goetz AE: Plateletendothelial cell interaction in pulmonary micro-circulation: the role of PARS. Thromb Haemost 2004;91:761-770.

26 Feuerhake F, Fuchsl G, Bals R, Welsch U: Expression of inducible cell adhesion molecules in the normal human lung: immunohistochemical study of their distribution in pulmonary blood vessels. Histochem Cell Biol 1998;110:387-394.

27 Kubo H, Doyle NA, Graham L, Bhagwan SD, Quinlan WM, Doerschuk CM: L- and P-selectin and CD11/CD18 in intracapillary neutrophil sequestration in rabbit lungs. Am J Respir Crit Care Med 1999;159:267-274.

28 Rinko LJ, Lawrence MB, Guilford WH: The molecular mechanics of P- and L-selectin lectin domains binding to PSGL-1. Biophys J 2004;86:544-554
29 Brunk DK, Hammer DA: Quantifying rolling adhesion with a cell-free assay: E-selectin and its carbohydrate ligands. Biophys J 1997; 72:2820-2833.

30 Greenberg AW, Brunk DK, Hammer DA: Cell-free rolling mediated by L-selectin and sialyl Lewis(x) reveals the shear threshold effect. Biophys J 2000;79:2391-2402.

31 Rodgers SD, Camphausen RT, Hammer DA: Sialyl Lewis(x)-mediated, PSGL-1-independent rolling adhesion on P-selectin. Biophys J 2000;79:694-706.

32 Parish CR: Heparan sulfate and inflammation. Nat Immunol 2005;6:861-862.

33 Wang L, Fuster M, Sriramarao P, Esko JD: Endothelial heparan sulfate deficiency impairs L-selectin- and chemokine-mediated neutrophil trafficking during inflammatory responses. Nat Immunol 2005;6:902-910.

34 Frenette PS, Moyna C, Hartwell DW, Lowe JB, Hynes RO, Wagner DD: Platelet-endothelial interactions in inflamed mesenteric venules. Blood 1998;91:1318-1324.

35 Kuklin V, Kirov M, Sovershaev M, Andreasen T, Ingebretsen OC, Ytrehus K, Bjertnaes L: Tezosentan-induced attenuation of lung injury in endotoxemic sheep is associated with reduced activation of protein kinase C. Crit Care 2005;9:R211-R217.

36 Tailor A, Granger DN: Hypercholesterolemia promotes $\mathrm{P}$-selectin-dependent platelet-endothelial cell adhesion in postcapillary venules. Arterioscler Thromb Vasc Biol 2003;23:675-680. 